\title{
Functional Dyeing and Finishing Using Catechins Extracted from Green Tea (II) - Evaluation of Anti-oxidant Activity of the Fabrics treated with Green Tea Extracts -
}

\author{
Songi Son, Kyungjin Jang, Taekyeong Kim, Jongsuc Jung ${ }^{1}$ and Younghee Choi ${ }^{2}$ \\ Dept. of Textile System Engineering, Kyungpook National University, Daegu, Korea \\ ${ }^{l}$ Dept. of Advanced Organic Material Science and Engineering, Kyungpook National University, Daegu, Korea \\ ${ }^{2}$ Shinpung Textile Co. Ltd., Daegu, Korea
}

(Received: September 9, 2008/Revised: September 23, 2008/Accepted: October 8, 2008)

\begin{abstract}
In order to introduce new functions onto textile fabrics, nylon and cotton were treated with green tea extracts.

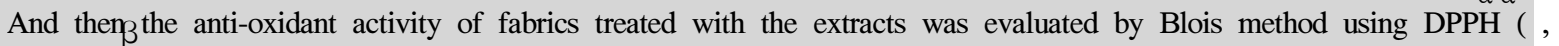
-diphenyl- -picrylhydrazyl). The anti-oxidant activity was exhibited on the textile fabrics treated as well as by the extracts itself. The cotton fabric treated with $10 \%$ o.w.f. of extract appeared to have almost $90 \%$ of anti-oxidant activity and $95 \%$ was obtained for nylon.
\end{abstract}

Keywords: green tea, catechins, anti-oxidant activity, polyphenol, dyeing, cotton, nylon

\section{Introduction}

Green teas are drawing considerable attention for specific health claims and the presence of functional constituents such as catechins. The catechins are composed of a family of four major substances, epicatechin (EC), epigallocatechin (EGC), epicatechin gallate (ECG), and epigallocatechin gallate (EGCG). These catechins have been reported for having many health benefits and functionality, such as anti-oxigenicity, anti-mutagenicity, anti-tumorgenicity and anti-carcinogenicity as well as decrease of cholesterol level in blood, prevention arterial sclerosis thrombosis and heart attack ${ }^{1-9)}$.

Recently, many creative technologies have been tried to introduce eco-friendly and health-care functions to textile products in textile field, too. One of those technologies is to use and apply the active ingredients obtained from natural materials, such as the catechins extracted from green tea ${ }^{10-13)}$.

In the previous study, green tea was extracted to be treated onto fabrics and the extracts were analyzed for its effective ingredients contents and their optimum treatment conditions toward fabrics were determined ${ }^{14,15}$.

$\mp$ In this study, two textile fabrics, nylon and cotton, were treated with green tea extracts at optimum conditions and investigated in terms of anti-oxidant activity of the fabrics, which was a representative function of green tea extracts, especially catechins.

\section{Experimental}

\subsection{Materials}

The green tea was purchased from Bosung, South Jeolla, Korea and was of grade used for a bath. Fabrics were $100 \%$ nylon and cotton, respectively. The other chemicals are reagent grade and used without further purification.

\subsection{Extraction of green tea}

Green tea $(50 \mathrm{~g})$ was extracted in distilled water $(1.0 \mathrm{~L})$ at 80 for 2 hours. The extracted solution was filtered and freeze-dried to obtain extracts powder $(3.5 \mathrm{~g})$ which was used in this experiment ${ }^{14,15}$.

\subsection{Treatment of green tea extracts onto fabrics}

Nylon and cotton fabrics $(1.0 \mathrm{\circ}$ ) were treated with green tea extracts (1 10\% o.w.f.) at 100 for 1 hour. 
Liquor ratio was 1:20 and $\mathrm{pH}$ was 5. This treatment conditions have been optimized in the previous study ${ }^{15}$. After treatment, the fabrics were washed with tap water for 5 minutes.

\subsection{Color measurement of treated fabrics}

The shade and color strength of treated fabrics were measured by a color measurement instrument (Spectrophotometer CM-3600d, Konica Minolta) and expressed by $L^{*} a^{*} b^{*} C^{*} h$ and total $\left.K / S\right)$. The total $K / S$ is the sum of the $K / S$ values in the visible region of the spectrum. Meanwhile, the $K / S$ is the color strength at single wavelength which is calculated from the reflectance $(R)$ of the single wavelength obtained at every $10 \mathrm{~nm}$ in the range of $400 \sim 700 \mathrm{~nm}$. The measurement was carried out with $10^{\circ}$ standard observer under standard light $\mathrm{D}_{65}$.

\subsection{Analysis of effective polyphenol content}

\subsubsection{Polyphenol content of green tea extracts}

Polyphenol content of the green tea extracts was calculated by UV-Vis. spectrophotometry based on FolinDenis method of $\mathrm{AOAC}^{16)}$. Folin reagent $(1 \mathrm{~mL})$ was added into the aqueous solutions of green tea extracts (1ml). After 3 minutes, 10\% sodium carbonate aquerous solution $(1 \mathrm{~mL})$ was added. The solution was kept at 30 for 1 hour in order to get endpoint of reaction between Folin reagent and polyphenol ingredients. And then the solution was measured to obtain absorbance increase at maximum absorbance wavelength, $766 \mathrm{~nm}$. By the calibration curve prepared previously using gallic acid as a standard reagent of polyphenols, the polyphenol contents of green tea extracts were calculated.

\subsubsection{Polyphenol content of the fabrics treated with extracts}

Polyphenol content of the treated fabrics was also obtained by the same method as 2.5 .1 . The fabrics $(0.2 \mathrm{~g})$ treated with appropriate amounts of green tea extracts were immersed in water $(2 \mathrm{~mL})$ and then Folin reagent $(2 \mathrm{~mL})$ was added. After 3 minutes, $10 \%$ sodium carbonate aqueous solution $(2 \mathrm{~mL})$ was added. After being kept at 3 0 for 1 hour, the solution was filtered and increased absorbance was measured at maximum absorbance wavelength, $766 \mathrm{~nm}$.

| 8 | www.ksdf.or.kr

\subsection{Evaluation of anti-oxidant activity (Electron Donating Ability, EDA)}

\subsubsection{Anti-oxidant activity of green tea extracts}

Anti-oxidant activity of the treated fabrics was obtained by UV-Vis. spectrophotometry based on Blois method using DPPH( , -diphenyl- -picrylhydrazyl)(Fig. 1).

The aqueous solutions of extracts $(4 \mathrm{ml})$ were mixed with $1.0 \mathrm{mM}$ DPPH ethanol solution $(1 \mathrm{~mL})$. The test solution was kept inside a darkroom at room temperature for 30 minutes in order to make the reaction complete between DPPH and polyphenol ingredients. And then absorbance decreases of the solution were measured at maximum absorbance wavelength, 517nm. Using the equation (1), anti-oxidant activity was calculated.

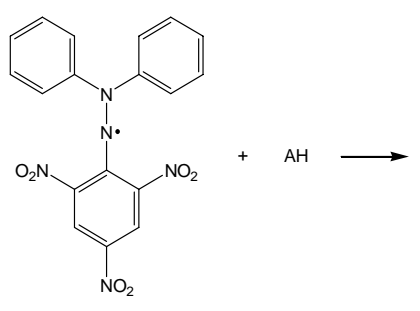

Diphenylpicrylhydrazyl (free radical, deep violet)

Fig. 1. Stabilization of DPPH radical by electron donation ability of anti-oxidants $(\mathrm{AH})$.

$\operatorname{EDA}(\%)=1-\frac{A_{1}}{A_{0}} \times 100$

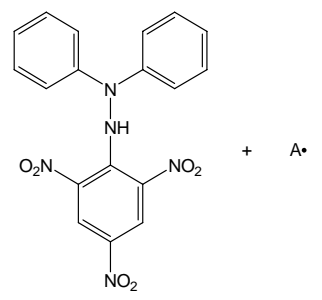

Diphenylpicrylhydrazine (nonradical, pale yellow)
EDA $(\%)=1-\frac{A_{1}}{A_{0}} \times 100$

$E D A$ : Electron donating ability (DPPH radical scav enging activity, Anti-oxidant activity)

$A_{0}$ : Absorbance of DPPH solution without sample at $517 \mathrm{~nm}$

$A_{1}$ : Absorbance of DPPH solution with sample at $517 \mathrm{~nm}$

\subsubsection{Anti-oxidant activity of the fabrics treated with extracts}

Anti-oxidant activity of the treated fabrics was obtained by the similar method as 2.6.2. The fabrics $(0.2 \mathrm{~g})$ treated with appropriate amounts of green tea extracts were immersed in 1.0mM DPPH ethanol solution ( $4 \mathrm{~mL})$. After the test solution was kept inside a darkroom at room temperature for 90 minutes, the solution was filtered and absorbance decrease was measured at maximum absorbance wavelength, $517 \mathrm{~nm}$. Using the equation (1), the anti-oxidant activity was calculated. 


\section{Results and Discussion}

\subsection{Treatment of nylon and cotton fabrics with green tea extracts}

Nylon and cotton fabrics were treated with green tea extracts at optimum conditions determined previously ${ }^{15}$. Fig. 2 shows the color strength values of the fabrics treated with different concentrations of green tea extracts. Nylon exhibited higher color strength than cotton fabrics, which is attributable to stronger polar interactions between protonated amino groups of nylon and hydroxyl groups of polyphenol ingredients in green tea extracts while only hydrogen bonding contributes between cotton and polyphenols ${ }^{15)}$.

Color values of the fabrics treated with $10 \%$ o.w.f. extracts were presented at Table 1 . Their color shades are yellowish brown.

\subsection{Analysis of green tea extracts}

\subsubsection{Polyphenol content of green tea extracts}

It is well known that the major effective ingredients of green tea are catechins that are classified into polyphenols. Therefore, it is necessary to analyze their content included green tea extracts.

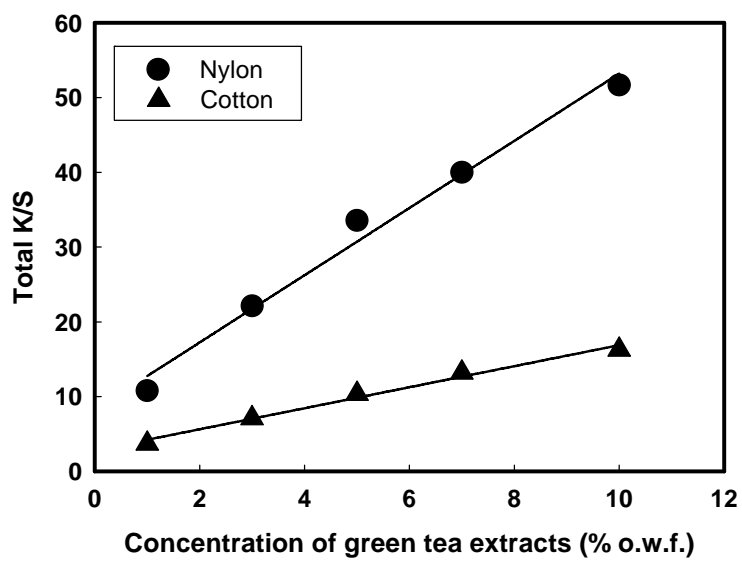

Fig. 2. Color strength of the fabrics treated with green tea extracts.

Fable 1 . Color values of the fabries treated $10 \%$ o.w.f. of green tea extracts

\begin{tabular}{cccccc}
\hline Fabric & $\mathrm{L}^{*}$ & $\mathrm{a}^{*}$ & $\mathrm{~b}^{*}$ & $\mathrm{C}^{*}$ & $\mathrm{~h}$ \\
Nylon & 55.2 & 9.6 & 20.5 & 22.7 & 64.9 \\
\hline Cotton & 69.6 & 7.1 & 14.6 & 16.3 & 64.0
\end{tabular}

In order to analyze the polyphenol content of green tea extracts, Foiln-Denis method was used. In this method, Folin reagent is used to detect polyphenols.

Without polyphenols, the test solution containing Folin reagent does not have any colors. By the existence of polyphenols, the Folin reagent solution changes its color into blue having a maximum absorption wavelength at $766 \mathrm{~nm}$ (Fig. 3).

The absorbances at $766 \mathrm{~nm}$ are linearly proportional to polyphenol concentration of the solution. By the calibration curve prepared previously using gallic acid as a standard reagent of polyphenols (Fig. 4), the polyphenol contents of green tea extracts were calculated.

A $0.1 \mathrm{~g} / 1$ of green tea extracts aqueous solution was prepared and analyzed by Folin-Denis method. As shown in Fig. 5, Absorbance of the solution at $766 \mathrm{~nm}$ was 0.516 which meant the green tea extracts contained $16 \%$ of polyphenols.

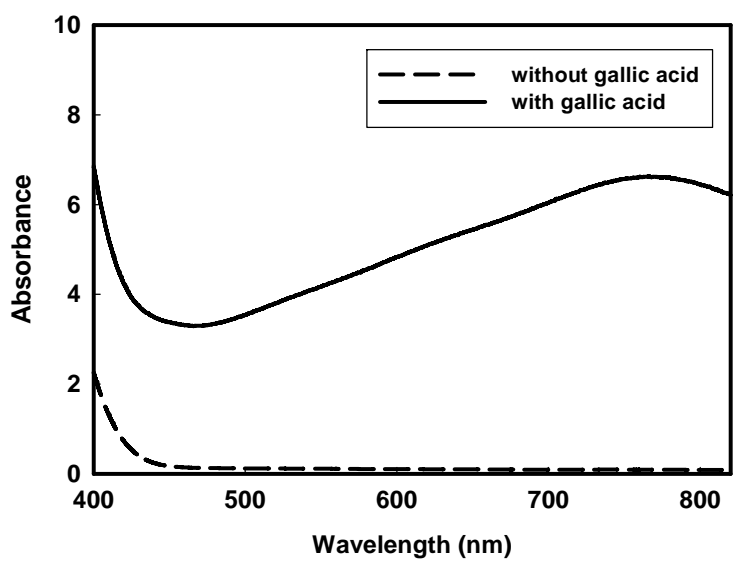

Fig. 3. Change of UV-VIS spectra of Folin reagent solution by polyphenol (gallic acid).

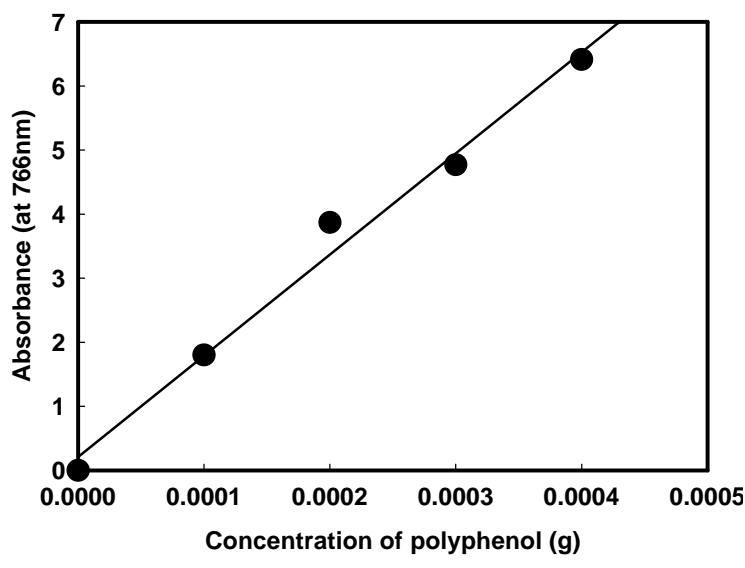

Fig. 4. Calibration curve of gallic acid by Folin reagent. J. of the Korean Soc. of Dyers and Finishers, Vol. 20, No. 5 | 9 | 


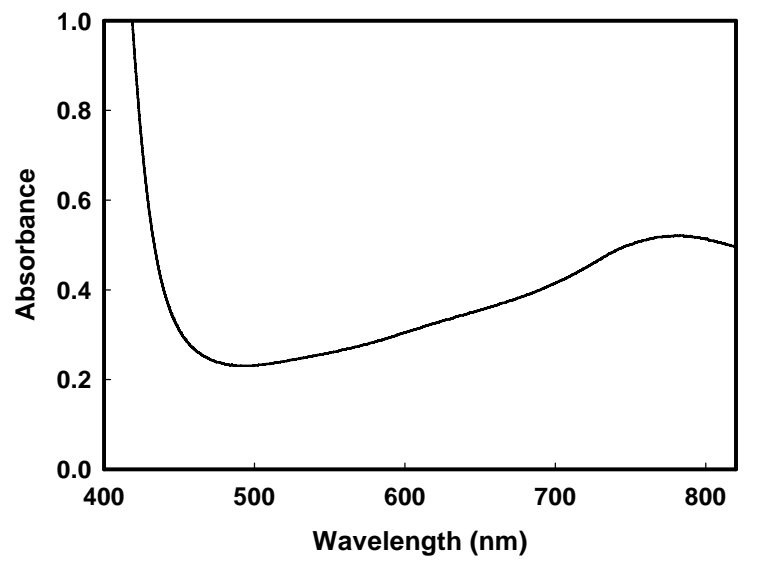

Fig. 5. UV-VIS spectrum of Folin reagent solution containing $0.1 \mathrm{~g} / \mathrm{l}$ of green tea extract solution.

\subsubsection{Anti-oxidant activity of green tea extracts}

The most well-known function of polyphenols is anti-oxidant activity. The activity means that the radical scavenging materials like polyphenols deactivate highly reactive and harmful species such as active oxygen radicals.

In order to evaluate the anti-oxidant activity of green tea extracts of this study, Blois method was used. In the method, DPPH could be used as a highly reactive radical like active oxygen to examine capability of electron donation of anti-oxidants like polyphenols in this experiment (Fig. 1). DPPH is a stable free radical and changes into non radical structure by electron donation from anti-oxidants. At this time, DPPH solution changes its color from deep violet into pale yellow as absorbance at the maximum absorption wavelength $(517 \mathrm{~mm})$ decreases (Fig. 1). The absorbance decrease is linearly proportional to concentration of anti-oxidants in the solution.

Fig. 6 shows the UV-Vis. spectra of DPPH solution containing different concentration of green tea extracts. The absorbances at $517 \mathrm{~nm}$ are decreasing continuously as the concentrations of green tea extracts are increasing. By using equation (1), electron donation ability(EDA), which means anti-oxidant activity, was calculated and showed in Fig. 7. For a comparison, BHA(butylated hydroxyanisole) and BHT(butylated hydroxytoluene), which are anti-oxidants being used for food, were investigated together. As the concentration of green tea extracts increased, EDA was also increased up to almost $80 \%$. The EDA of extracts seems comparable to those of BHA and BHT.

| 10 | www.ksdf.or.kr

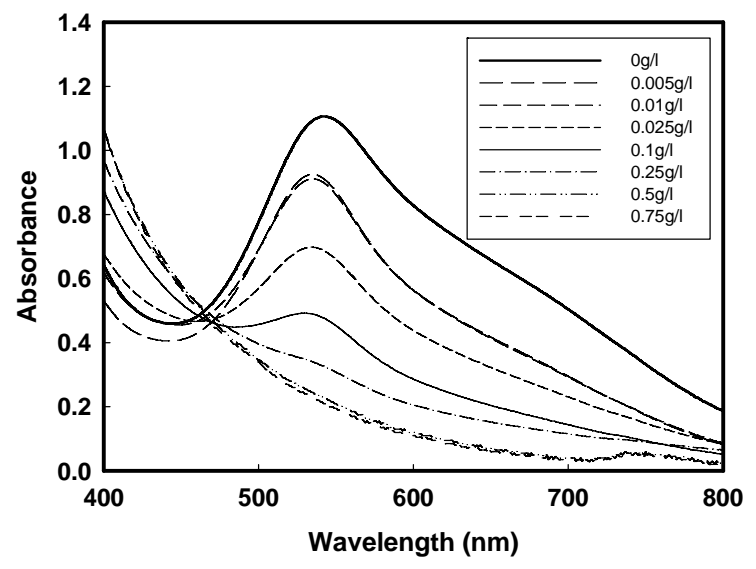

Fig. 6. UV-VIS spectra of DPPH solution containing various concentration of green tea extracts.

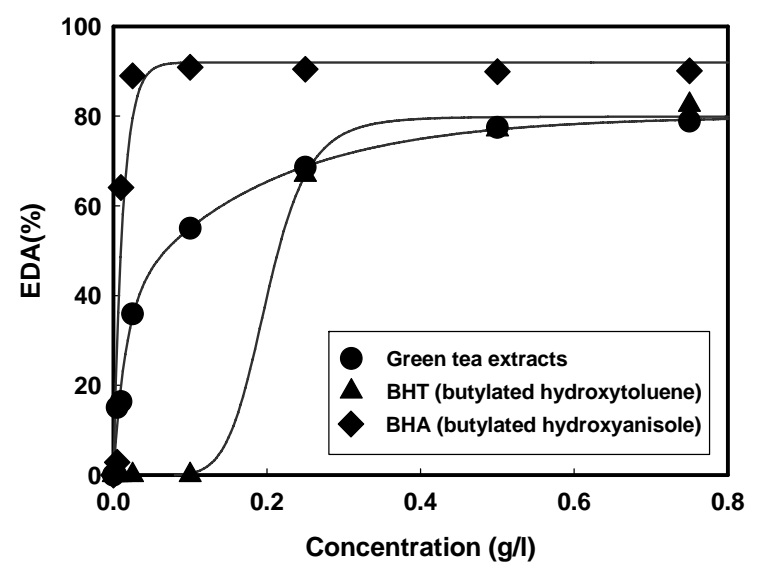

Fig. 7. EDA (anti-oxidant activity) of green tea extracts.

\subsection{Analysis of the fabrics treated with green tea extracts}

\subsubsection{Effective polyphenol content of the fabrics treated with green tea extracts}

Fig. 8 shows the absorbance increase of Folin reagent solutions containing fabrics treated with green tea extracts. From the results of Fig. 8, the effective polyphenol content of nylon and cotton fabrics treated with green tea extract was calculated and presented in Fig. 9. The effective polyphenol content of cotton fabrics was higher than that of nylon. This seems to be inconsistence with the result of Fig. 2 that showed higher uptake of green tea extracts onto nylon than onto cotton fabrics. This could be explained as follows; Since the attraction of green tea extracts toward nylon fibers is stronger than toward cotton, nylon has much higher uptake of extracts than cotton. However, the stronger attraction onto nylon would adversely affect anti-oxidant activity, because in order for 
anti-oxidants to act toward reactive radicals, the antioxidants should be loose out from the fibers and participate to radical scavenging reactions. Moreover, it is needed to comment that the effective polyphenol content of the fabrics is not exactly the same as polyphenol uptake
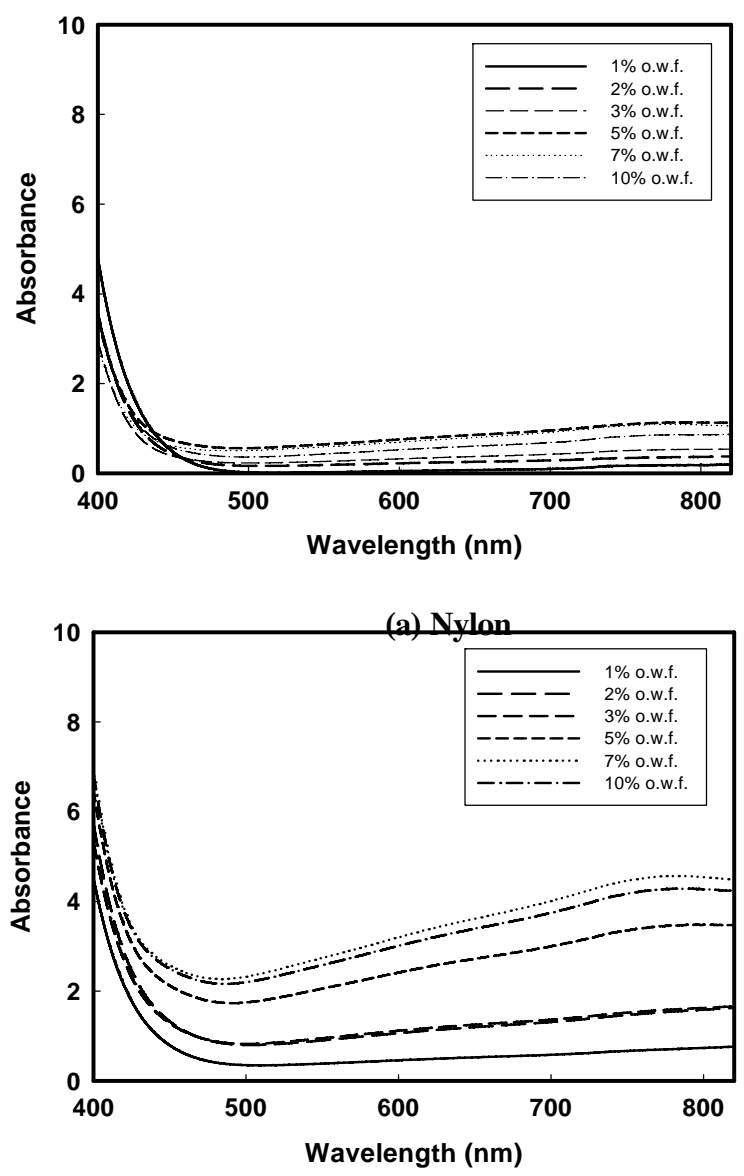

(b) Cotton

Fig. 8. UV-VIS spectra of Folin reagent solution nnntainins tho fahrinc trostad waith aroon tos ovtronts.

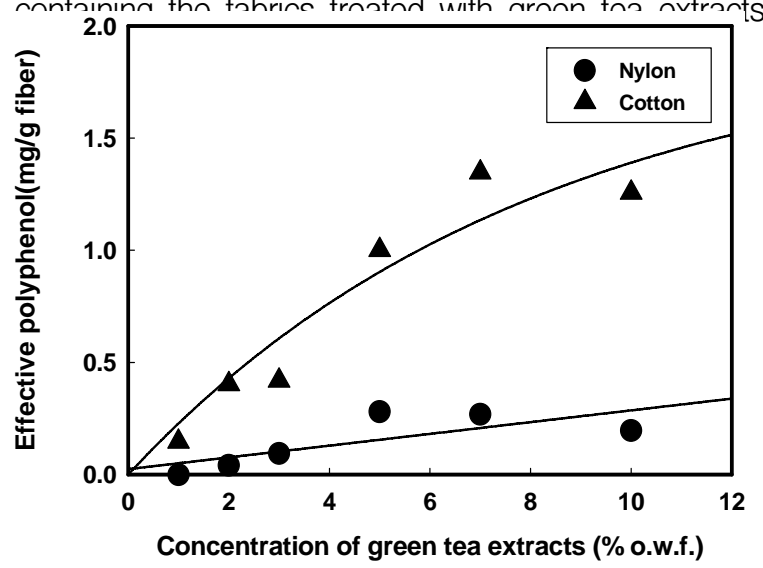

Fig. 9. Effective polyphenol content of the fabrics treated with green tea extracts. on the fabrics. This is because all polyphenols inside the fabrics might not participate to the reaction with Folin reagent.

\subsubsection{Anti-oxidant activity of the fabrics treated with green tea extracts}

Fig. 10 shows the absorbance decrease of DPPH solutions containing fabrics treated with green tea extracts. From the results of Fig. 10, the anti-oxidant activity of nylon and cotton fabrics treated with green tea extract was calculated and presented in Fig. 11. Both nylon and cotton fabrics exhibited considerable anti-oxidant activity. But the values of cotton were somewhat higher than those of nylon. The cotton fabric treated with $10 \%$ o.w.f. of extract appears to have almost $90 \%$ of anti-oxidant activity and $95 \%$ was obtained for nylon. This result means that the useful active ingredients like polyphenols might exhibit medical actions toward human body by contact with textile fabrics as well as by intake from food or by spreading on the skin.
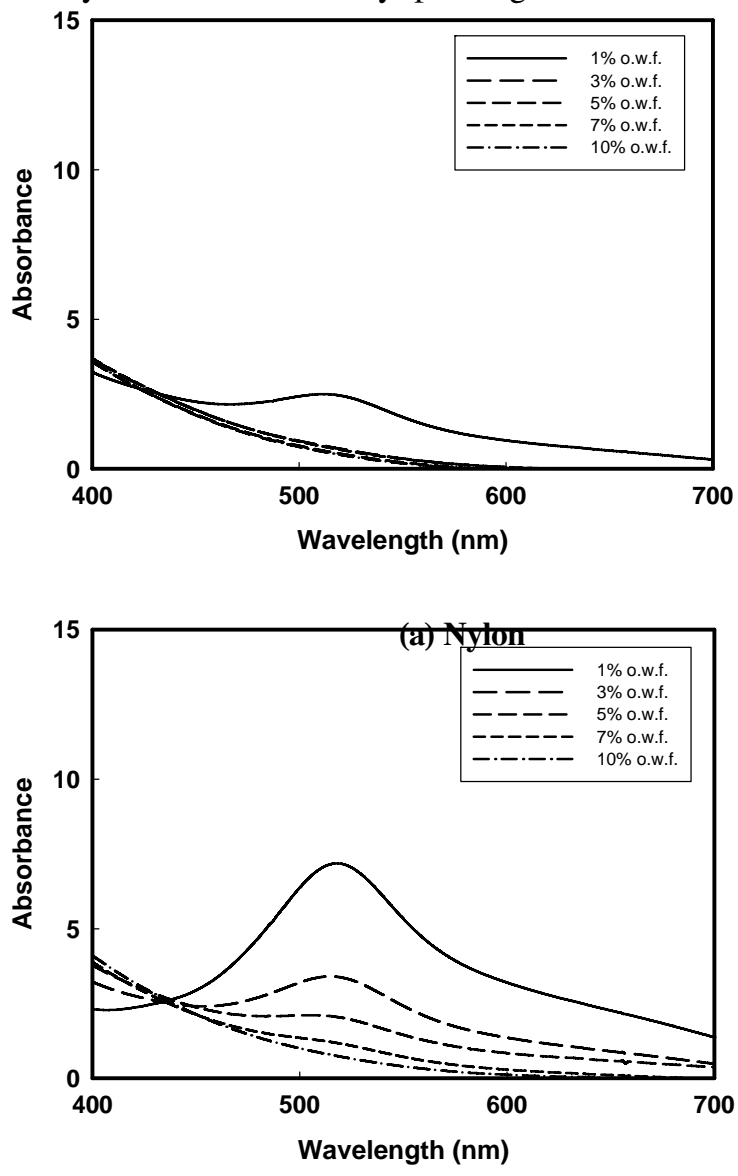

(b) Cotton

Fig. 10. UV-VIS spectra of DPPH solutions containing the fabrics treated with green tea extracts.

J. of the Korean Soc. of Dyers and Finishers, Vol. 20, No. 5 | 11 


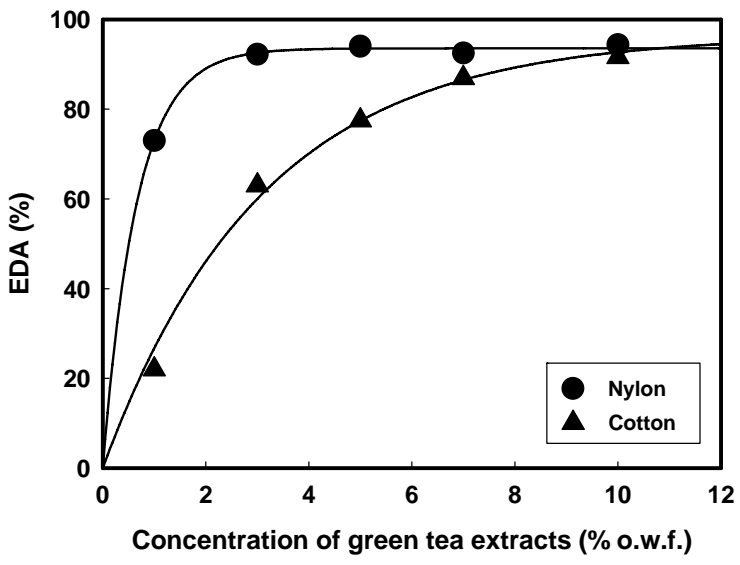

Fig. 11. EDA (anti-oxidant activity) of the fabrics treated with green tea extracts.

\section{Conclusion}

In order to examine whether the active ingredients of green tea extracts can be utilized for textile fabrics and whether a new functional activity can be introduced to fabrics, nylon and cotton were treated with green tea extracts. As results, an anti-oxidant activity was exhibited not only by the extracts itself but also on the fabrics showing $90 \%$ of EDA for cotton and $95 \%$ for nylon fabrics.

In the past, anti-oxidant activity was considered and investigated only for food and cosmetics. However, this kind of activity could be a new function of textile product and will be more and more important in the future.

In addition, it should be commented that many additional experimental results should be supplemented in the medical fields as well as textile fields to prove its positive effect to human body.

\section{References}

1. Y. H. Park, Effect of PH on the Stability of Green Tea Catechins, Journal of Food Hygiene and Safety, 17(3), 117(2002).

2. J. H. Lee, Y. M. Lee, and D. C. Moon, Rapid Separation and Identification Method of Tea Catechins, Journal of the Korean Society of Analytical Sciences, 5(3), 333-338(1992).

3. Y. P. Yun, W. S. Kang, and M. Y. Lee, The Antithrombotic Effects of Green Tea Catechins, Journal of Food Hygiene and Safety, 11(2), 77-82 (1996).

4. Y. J. Lee, M. S. Ahn, and W. T. Oh, A Study on | 12 | www.ksdf.or.kr the Catechins Contents and Antioxidative Effect of Various Solvent Extracts of Green, Oolong and Black Tea, Journal of Food Hygiene and Safety, 13(4), 370-376(1998).

5. J. I. Kim and K. H. Row, Recovery of Catechin Compound from Korean Green Tea by Solvent Extraction and Partition, Korean Journal of Biotechnology and Bioengineering, 16(5), 442-445(2001).

6. K. H. Row and Y. Jin, Recovery of Catechin Compounds from Korean Tea by Solvent Extraction, Bioresource Technology, 97, 790-793(2006).

7. C. J. Chang, K. L. Chiu, Y. L. Chen, and C. Y. Chang, Seperation of Catechins from Green Tea Using Carbon Dioxide Extraction, Food Chemistry, 68, 109-113(2000).

8. A. P. Uzunalic, M. Skerget, Z. Knes, B. Weinreich, F. Otto, and S. Gruner, Extraction of Active Ingredients from Green Tea : Extraction Efficiency of Major Catechins and Caffeine, Food Chemistry, 96, 597-605(2006).

9. Y. Yoshida, M. Kiso, and T. Goto, Efficiency of the Extraction of Catechins from Green Tea, Food Chemistry, 67, 429-433(1999).

10. J. H. Son, M. S. Lee, and T. I. Chun, Catechins Content and Color Values of Silk Fabrics Dyed with Korean Green Tea Extracts, Journal of the Korean Society of Dyers and Finishers, 18(1), 10-19 (2006).

11. Y. S. Shin and H. Choi, Characteristics and Dyeing Properties of Green Tea Colorants (Part I) - Components and Characteristics of Green Tea Colorants, Journal of the Korean Society of Clothing and Textiles, 23(1), 140-146(1999).

12. Y. S. Shin and H. Choi, Characteristics and Dyeing Properties of Green Tea Colorants (Part II) Dyeing Properties of Silk with Green Tea Colorants, Journal of the Korean Society of Clothing and Textiles, 23(3), 385-390(1999).

13. Y. S. Shin and H. Choi, Characteristics and Dyeing Properties of Green Tea Colorants (Part III) - Dyeing Properties of Cotton with Green Tea Colorants, Journal of the Korean Society of Clothing and Textiles, 23(4), 510-516(1999).

14. T. K. Kim, S. I. Son, Jongsuc Jung, Kyungjin Jang, Ohkyung Kwon, Younghee Choi, and Young-Han 
Jeong, Functional Dyeing and Finishing using Catechins Extracted from Grean Tea (Part ${ }^{\mathrm{I}}$ ) Extraction Optimization, Stability, and Content Analysis of Catechins, Journal of the Korean Society of Dyers and Finishers, 20(2), 75-82(2008).

15. S. I. Son, Kyungjin Jang, T. K. Kim, Jongsuc Jung, and Younghee Choi, Functional Dyeing and Finishing using Catechins Extracted from Grean Tea - Dyeing
Optimization and Fastness, Journal of the Korean Society for Clothing Industry, submitted.

16. S. K. Lee, H. J. Lee, M. H. Yu, H. G. Im and I. S. Lee, Total Polyphenol Contents and Antioxidant Activities of Methanol Extracts from Vegetables produced in Ullung Island, Journal of the Korean Society of Food Science and Technology, 37(2), 233-240(2005). 